Progress in Flight Physics 5 (2013) 125-138

DOI: 10.1051/eucass/201305125

(C) Owned by the authors, published by EDP Sciences, 2013

\title{
A SUMMARY OF LASER AND MICROWAVE FLOW CONTROL IN HIGH-SPEED FLOWS
}

\section{Knight}

\author{
Rutgers, The State University of New Jersey
}

New Brunswick, New Jersey 08903, USA

\begin{abstract}
Laser and microwave discharge in air has emerged as an effective method for flow control in high-speed flows. Computational and experimental research has demonstrated its capability for significant drag reduction and mitigation of adverse interactions in high-speed flows. The paper presents a summary of key computational and experimental studies performed at Rutgers University in collaboration with the Joint Institute for High Temperatures (Moscow, Russia) and St. Petersburg State University (St. Petersburg, Russia).
\end{abstract}

\section{INTRODUCTION}

The design of effective high-speed air vehicles necessarily includes careful consideration of flow control issues. In this context, flow control includes both mitigation (or avoidance) of potentially deleterious flow situations (e.g., high heat transfer associated with reattaching shear layers) and improved aerodynamic performance (e.g., drag reduction).

Conventional electromechanical devices for flow control (e.g., surface jets, boundary layer bleed, etc.) lose effectiveness with increasing Mach number due to their inherent inertia. Typical actuation times (i.e., the time interval necessary to fully actuate the device) are tenths to hundredths of seconds. However, the flowfield timescale for a high-speed vehicle (e.g., the representative length scale of the vehicle divided by its velocity) is in the range of milliseconds (e. g., a 10meter vehicle traveling at Mach 6.8 at 20-kilometer altitude).

Laser and microwave discharge have emerged as effective methods for flow control in high-speed flows. Such discharges create a high-temperature plasma in the flow which interacts with the wave structure of a high-speed vehicle, thereby changing the flow patterns and aerothermodynamic loads. Laser and microwave discharges have actuation times measured in a few nanoseconds to a microsecond which are orders of magnitude smaller than conventional electromechanical devices.

This is an Open Access article distributed under the terms of the Creative Commons Attribution License 2.0, which permits unrestricted use, distribution, and reproduction in any medium, provided the original work is properly cited. 
A growing body of computational and experimental evidence supports the concept of laser and microwave discharge for flow control. The field of energy deposition for flow control extends beyond laser and microwave discharge, and the reader is referred to additional references for further information [1-6].

This paper presents a summary of three research projects on laser and microwave energy deposition for flow control performed at Rutgers University. The microwave research is in collaboration with the Joint Institute for High Temperatures (Moscow, Russia) and St. Petersburg State University (St. Petersburg, Russia). The results of these projects have previously been published in separate papers, and it was, therefore, concluded that a summary compendium of the results would be useful.

\section{LASER AND MICROWAVE DISCHARGE IN AIR}

Pulsed laser discharge in a gas was discovered by Damon and Tomlinson [7] and Minck [8]. A detailed discussion is presented in [9] and summarized in Fig. 1. The incident laser radiation generates seed electrons by multiphoton ionization (Fig. 1a) followed by cascade release of electrons (Fig. 1b). A detonation wave forms in the plasma and propagates along the beam axis towards the laser source (Fig. 1c). Upon completion of the laser pulse, the detonation wave terminates and the heated high temperature and high pressure plasma generates a blast wave which propagates outwards (Fig. 1d). A toroidal vortex forms due to the baroclinic instability associated with the detonation wave. Notwithstanding the complex thermochemistry and asymmetry of the plasma thus formed, once the blast wave has propagated significantly beyond the plasma, the gas dynamic effect of the laser discharge is (to the first approximation) the formation of a roughly spherical region of high-temperature gas. In particular, when the laser discharge occurs in a supersonic flow, the timescale for formation of the plasma is typically several orders of magnitude smaller than the relevant timescales of the flowfield (e.g., $L / U_{\infty}$ where $L$ is the suitable length scale of an aerodynamic body such as an airfoil chord, and $U_{\infty}$ is the freestream velocity), and, therefore, to the first approximation, the laser discharge generates a heated region of roughly spherical shape at the same pressure as the ambient and traveling with the same velocity of the surrounding fluid. Consequently, the laser 'spot' has a Mach number which is less than the surrounding flow that constitutes a principal parameter determining its effect on the shock structure of the aerodynamic body.

Pulsed microwave discharge is considerably more variable in structure depending upon the ambient pressure and the properties of the microwave field (i. e., microwave wavelength, electric field intensity, circular vs. linear polarization) as indicated in Fig. 2. The initial microwave pulse accelerates the ambient electrons resulting in cascade ionization. The electrons collide elastically and 


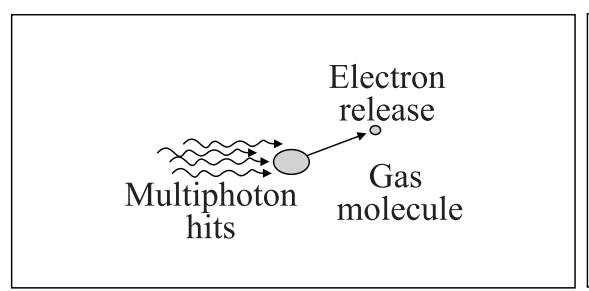

(a)

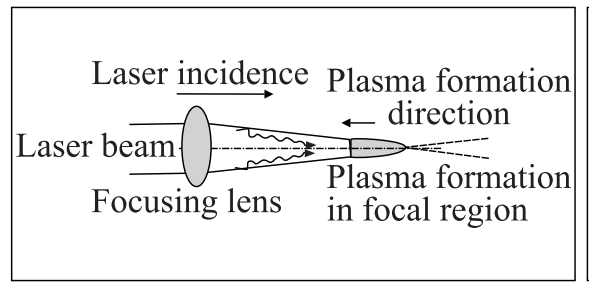

(c)

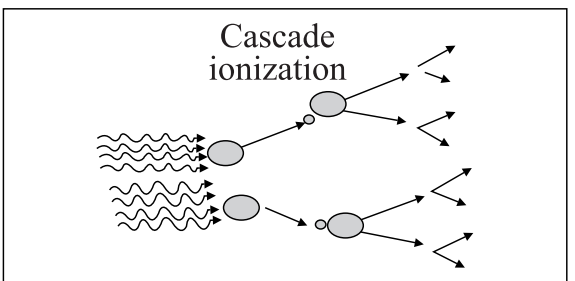

(b)

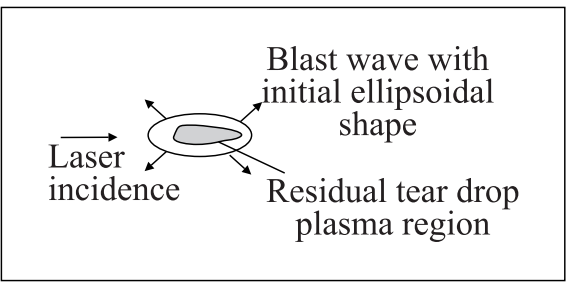

$(d)$

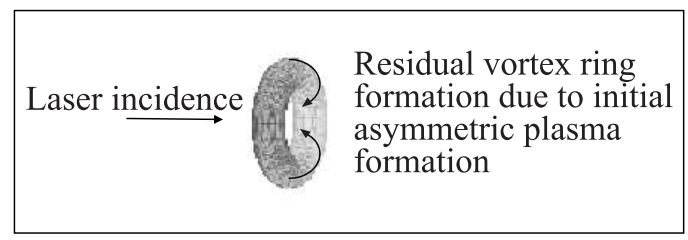

$(e)$

Figure 1 Laser discharge [10]

inelastically with the heavy particles. The elastic collisions have negligible effect on the kinetic energy of the heavy particles due to the difference in mass of approximately $10^{-5}$. The inelastic collisions provide energy transfer through several different mechanisms including rotational excitation, vibrational excitation, excitation of electronic states, dissociations, and ionizations. The three main kinetic paths for energy transfer from these modes to the kinetic energy of the heavy particles (i. e., increasing the gas temperature) are rotational excitation, collisional quenching of the excited states, and electron-ion recombination [12].

\section{LASER DISCHARGE FOR DRAG REDUCTION}

Adelgren et al. [10, 13, 14] investigated experimentally the effect of a 10nanosecond pulsed Nd:Yag $(532 \mathrm{~nm}$ ) laser discharge on flow past a hemisphere of diameter $D$ at Mach 3.45 in air at $\operatorname{Re}_{D}=1.7 \cdot 10^{6}$. Figure 3 displays a time 


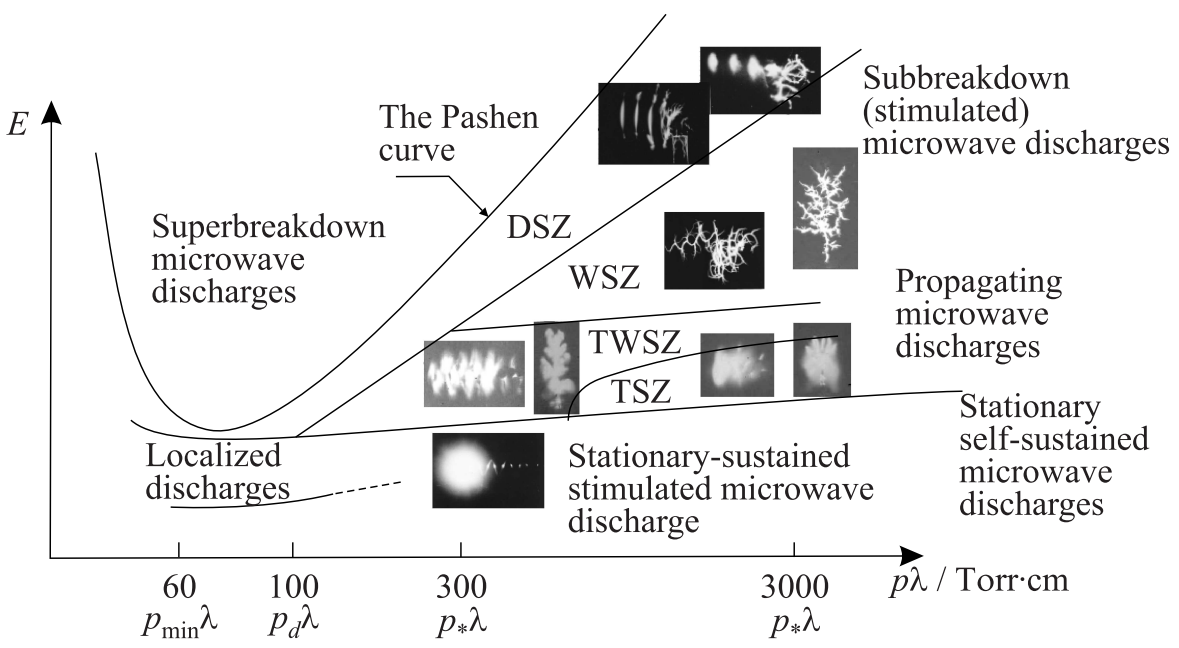

Figure 2 Examples of microwave discharge [11]

sequence of Schlieren images for a pulse energy of $258 \mathrm{~mJ}$ focused one diameter upstream of the stagnation point of the hemisphere. The focus of the laser pulse is indicated by the bright spot* in Fig. $3 a$. The blast wave impacts the hemisphere before the laser-generated plasma reaches the blunt-body shock and reflects from the hemisphere surface resulting in a momentary rise in the surface pressure. Figure $4 a$ displays the surface pressure vs. time on the centerline of the sphere for pulse energies of 13,127 , and $258 \mathrm{~mJ}$. The magnitude of the initial compression increases with increasing pulse energy as expected. Upon intersection of the plasmoid with the blunt-body shock (see Fig. $3 c$ ), the blunt-body shock lenses forward (see, for example, [15]) and a toroidal recirculation region momentarily forms creating an effective aerodynamic streamlining. The lower Mach number in the plasmoid (relative to the freestream) implies a lower stagnation pressure and, hence, the stagnation point is momentarily moved off the hemisphere centerline to a position ahead of the hemisphere. It is interesting to note that the minimum pressure is effectively independent of the pulse energy despite an approximately twenty-fold difference.

Figure $4 b$ displays the surface pressure on the entire windward hemisphere surface vs. time. The initial compression due to the intersection of the blast wave with the hemisphere is evident, followed by the expansion associated with the formation of the toroidal vortex and the recompression and relaxation to the steady condition.

${ }^{*}$ Due to saturation of the CCD (charge-coupled device) camera, this spot persists although the laser-generated plasma ("plasmoid") is convected downstream. 


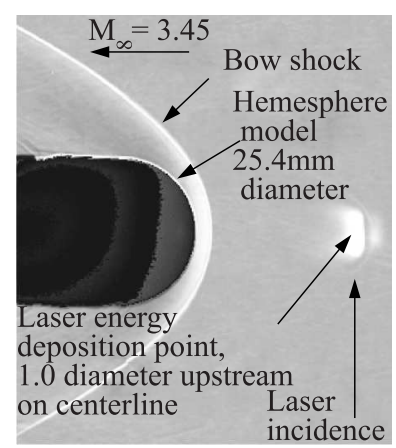

(a)

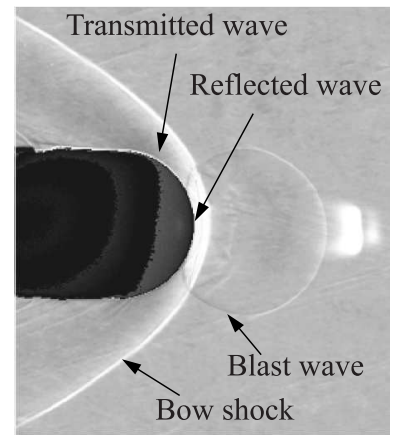

(b)

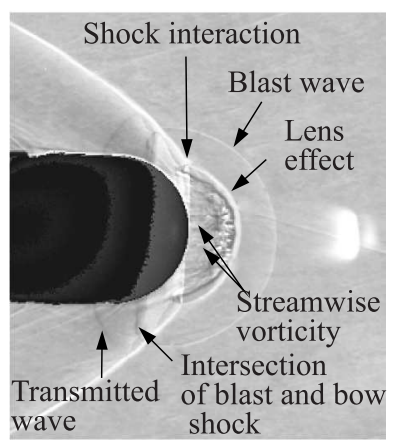

(c)

Figure 3 Interaction of laser discharge and hemisphere for 283-millijoule pulse [10, 13, 14]: $(a) t=0 \mu \mathrm{s} ;(b) 30$; and (c) $t=50 \mu \mathrm{s}$

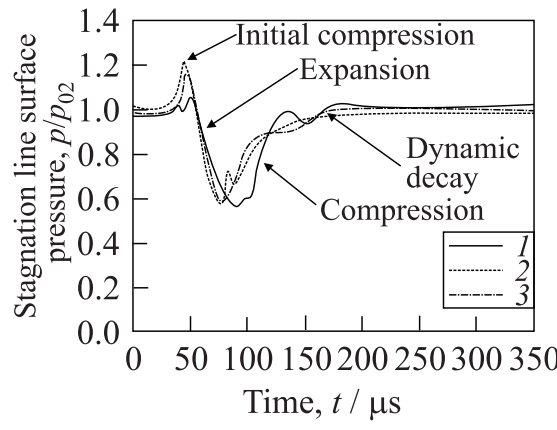

(a)

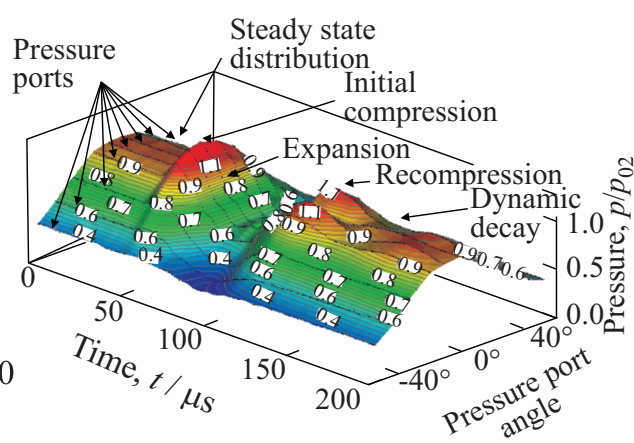

(b)

Figure 4 Surface pressure vs. time $[10,13,14]$ : (a) 13- (1), 127- (2), and 258millijoule (3) pulses; and (b) 127-millijoule pulse. 


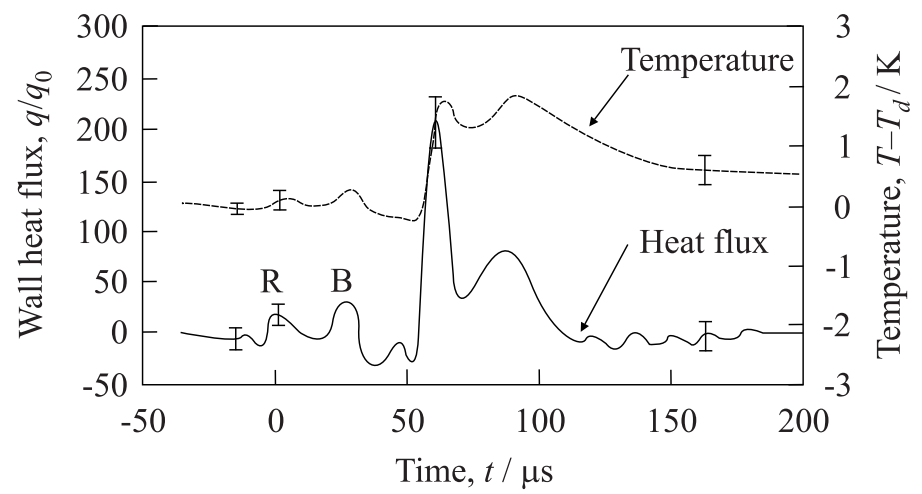

(a)

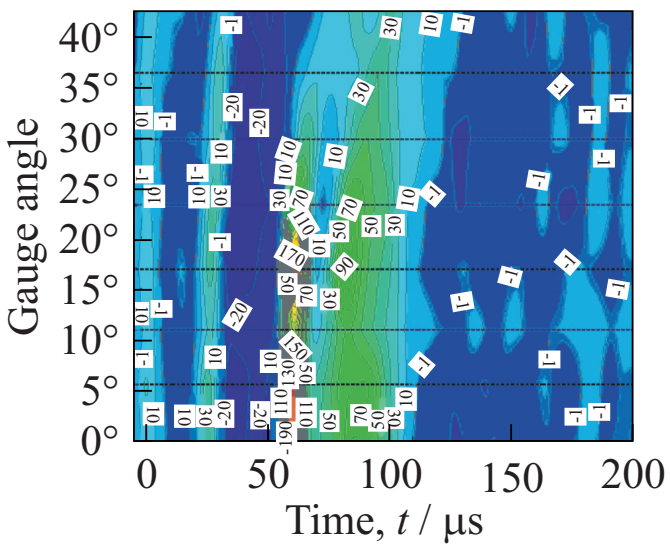

(b)

Figure 5 Surface heat transfer vs. time for 283-millijoule pulse [10, 13]: (a) heat transfer on centerline; and $(b)$ heat transfer on surface.

Figure 5 displays the surface heat transfer vs. time on the centerline (Fig. $5 a$ ) and on the surface (Fig. $5 b$ ). The interaction of the plasmoid with the blunt-body shock generates a momentary increase in heat transfer at the surface.

\section{LASER DISCHARGE FOR EDNEY IV INTERACTION}

Adelgren et al. $[10,13,14]$ investigated experimentally the effect of a 10nanosecond pulsed Nd:Yag (532 nm) laser discharge on an Edney IV interac- 


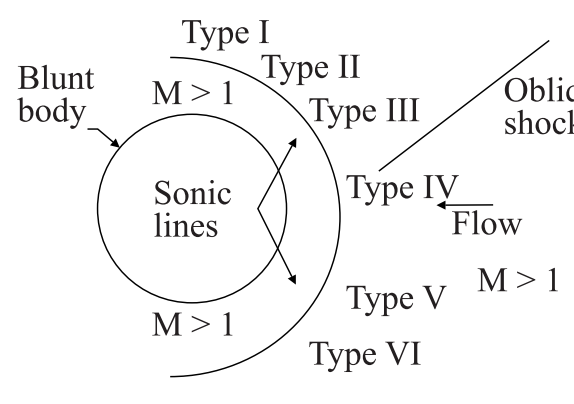

(a)

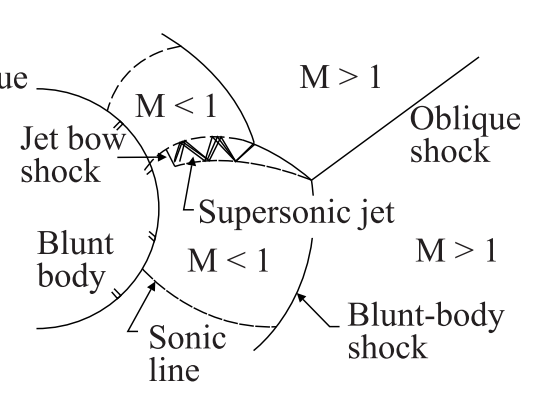

(b)

Figure 6 Shock-shock interactions [16]: (a) Edney I-VI; and (b) Edney IV

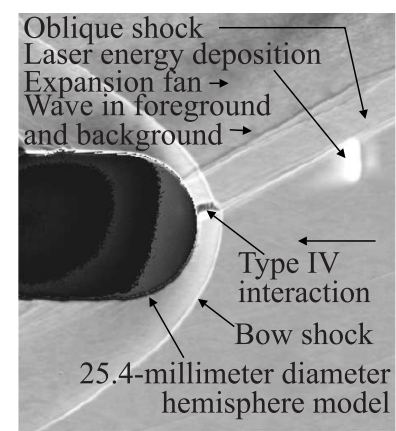

(a)

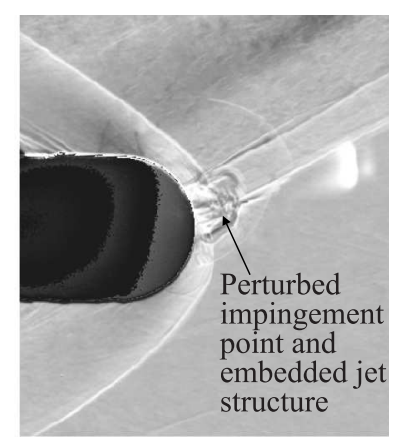

(b)

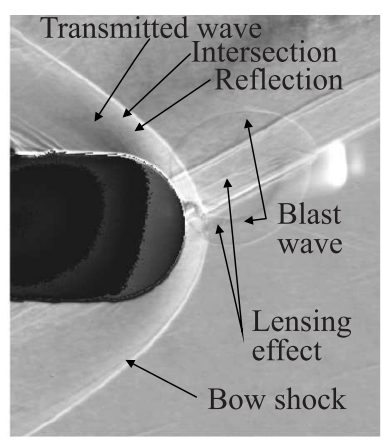

(c)

Figure 7 Intersection of laser discharge and Edney IV for $283 \mathrm{~mJ}$ pulse [10]: $(a) t$ $=0 \mu \mathrm{s} ;(b) 30$; and $(c) t=50 \mu \mathrm{s}$ 
tion at Mach 3.45 and $\operatorname{Re}_{D}=1.8 \cdot 10^{6}$. The Edney IV interaction [16] is one of several different types of shock-shock interactions involving a blunt-body shock and an impinging shock. The classification of the six major types of Edney interactions is displayed in Fig. $6 a$ and the particular configuration of the Edney IV interaction is illustrated in Fig. $6 b$. The impingement of the supersonic jet, formed by the Edney IV interaction, on the surface may result in heat transfer

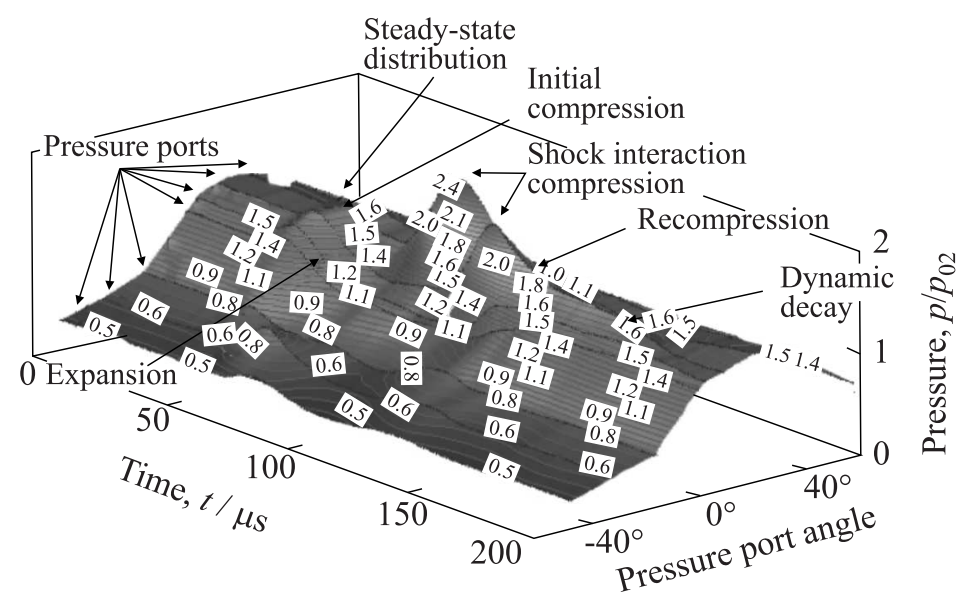

(a)

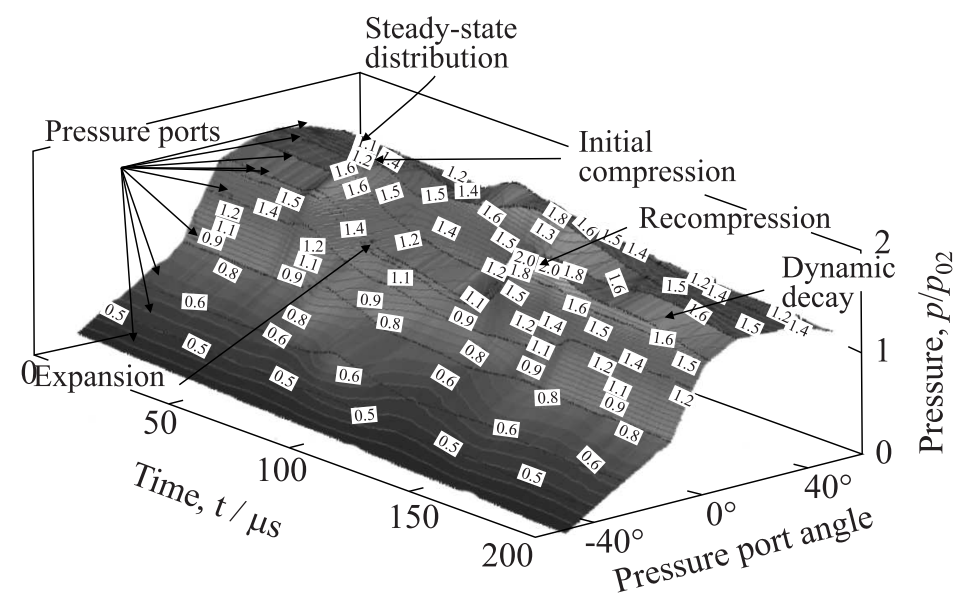

(b)

Figure 8 Surface pressure vs. time for 127-millijoule pulse [10, 13, 14]: $(a)(x, r)$ $=(-0.7 D, 0.15 D) ;$ and $(b)(x, r)=(-0.7 D, 0.28 D)$ 
several orders of magnitude higher than ordinary stagnation point heat transfer [17]. Figure 7 displays a sequence of schlieren images for the interaction of a 283-millijoule pulse with the Edney IV interaction due to the intersection of an oblique shock (generated by a separate ramp) with the blunt-body shock of a hemisphere. The focus of the discharge is $1.0 D$ upstream of the hemisphere and $0.45 \mathrm{D}$ above the centerline. The focal location of the laser pulse is indicated by the bright spot. The impingement of the blast wave on the hemisphere is indicated in Fig. $7 b$. The interaction of the plasmoid with the Edney IV interaction significantly alters the shock-shock interaction momentarily leading to a change in surface pressure distribution.

The effect of the focal location of the laser pulse is shown in Fig. 8. Figure $8 a$ shows the pressure vs. time on the hemisphere surface for a focal position $0.7 D$ upstream of the hemisphere and $0.15 D$ above the centerline. An initial compression is followed by an expansion and a subsequent overpressure associated with the shock-shock interaction. Figure $8 b$ shows the pressure vs. time on the hemisphere surface for a focal position $0.7 D$ upstream of the hemisphere and $0.28 D$ above the centerline. In contrast to Fig. $8 a$, no overpressure is observed and a momentary pressure drop up to $30 \%$ is achieved. Further research is needed to identify the optimal focal position of the laser pulse to achieve maximum momentary pressure decrease (and, by analogy, presumably maximum momentary decrease in surface heat transfer).

\section{MICROWAVE DISCHARGE FOR DRAG REDUCTION}

Knight et al. $[12,18]$ investigated computationally the effect of a 1.2-microsecond microwave pulse on the flow past a hemisphere cylinder at Mach 2.1 correspond-

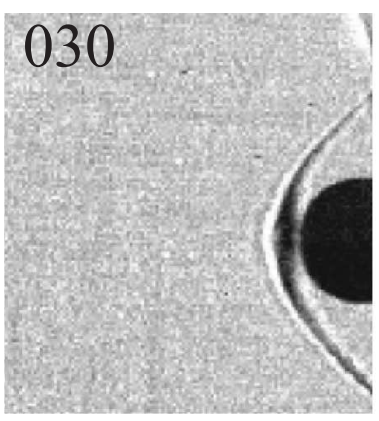

(a)

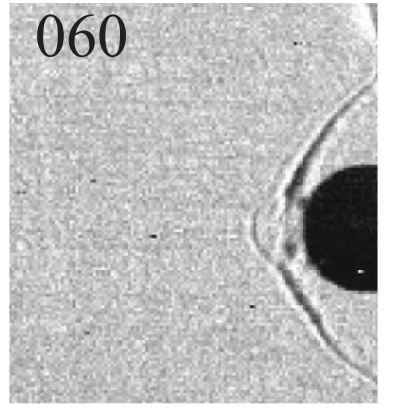

(b)

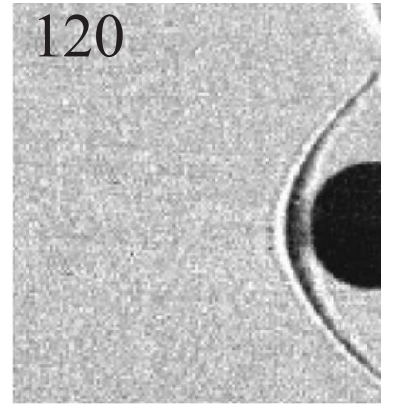

(c)

Figure 9 Interaction of microwave discharge and hemisphere [19]: $(a) t=0 \mu \mathrm{s} ;(b) 60$; and $(c) t=120 \mu \mathrm{s}$. The number in the upper left corner is the time in microseconds subsequent to the microwave pulse 


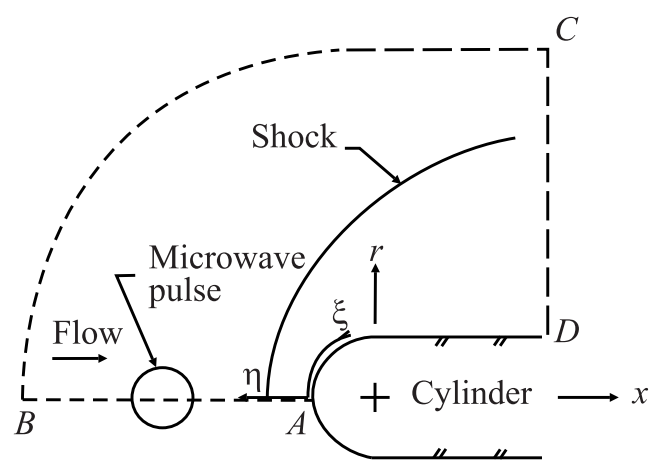

Figure 10 Domain [12]

ing to the experiments of Lashkov et al. [19]. Experimental schlieren images of the interaction of the 9-gigahertz microwave-generated plasma, initially focused upstream of the hemisphere cylinder, with the blunt-body shock are displayed in Fig. 9. The interaction of the heated plasma with the blunt-body shock results in a lensing forward of the shock and formation of a toroidal vortex (Fig. 9b). After a sufficient time, the flow relaxes to its undisturbed state (Fig. 9c).

The computations were performed using a gas dynamic code with a thermochemistry model for the microwave-generated plasma incorporating 23 species and 238 reactions. Details of the code are presented in [18]. The computational domain and focus of the microwave pulse are shown in Fig. 10.

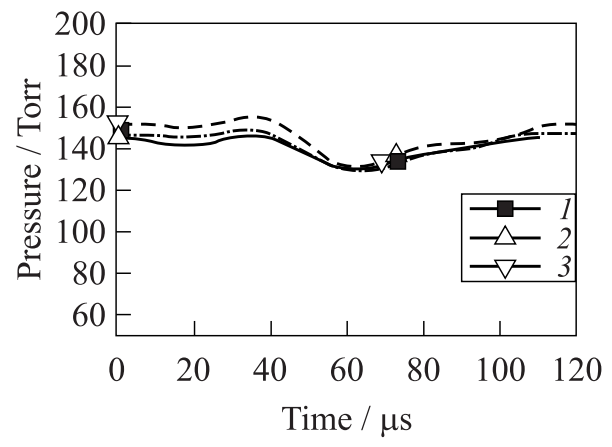

Figure 11 Dependence $p$ vs. $t$ [12]: 1 - experiment; 2 - computations (Grid No. 1); and 3 -computations (Grid No.2). The computed surface pressure is filtered in time to reflect the frequency response characteristics of the experimental surface pressure transducer [18] 


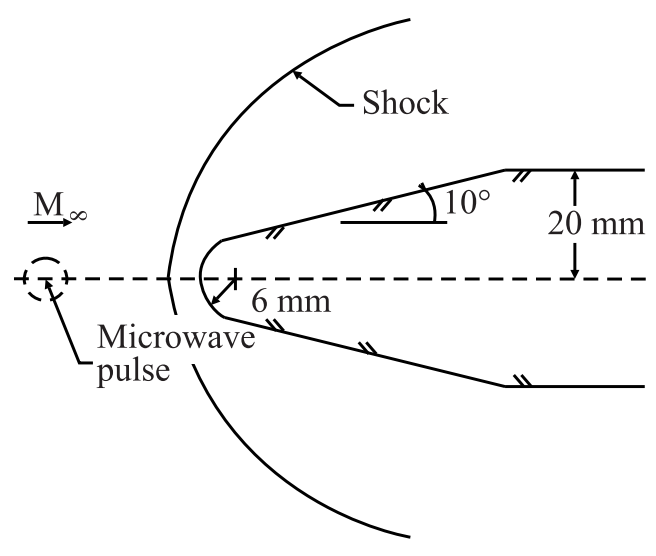

Figure 12 Domain [20]

The computed and experimental surface pressure on the hemisphere centerline are displayed in Fig. 11. Good agreement between the computation and experiment is evident, and the interaction of the microwave-generated plasmoid results in a momentary drag reduction on the hemisphere cylinder.

Knight et al. [20] also considered the interaction of a microwave-generated plasma with a hemisphere-cone-cylinder at Mach 2.1. The flowfield configuration is illustrated in Fig. 12.

The initial condition prior to the microwave discharge is the converged computed flowfield past the hemisphere-cone cylinder shown in Fig. 13. The computations were performed for two different assumed axial lengths of the imposed pulsed electric field $l_{f}=0.5(n+1) \lambda$ where $n=0$ or 1 and $\lambda$ is the microwave

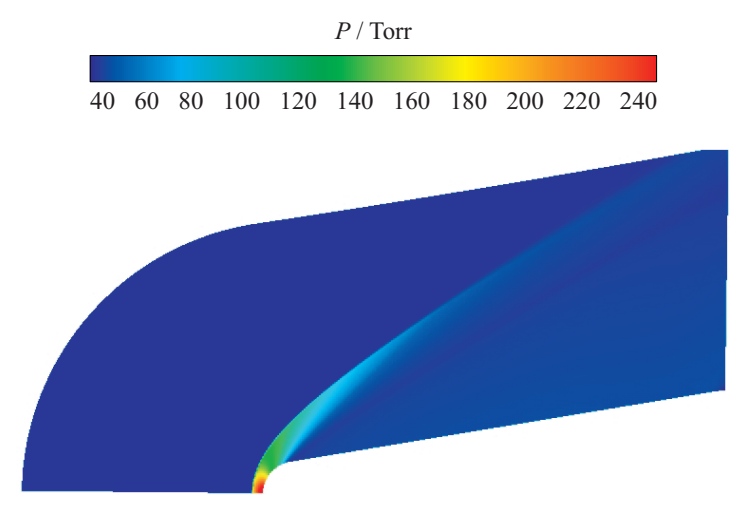

Figure 13 Initial condition pressure contours [20]. 


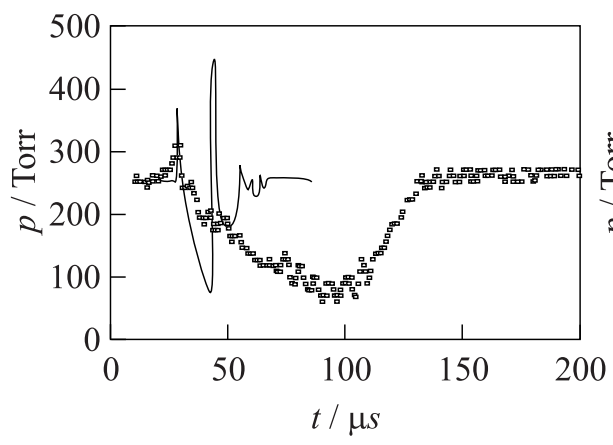

(a)

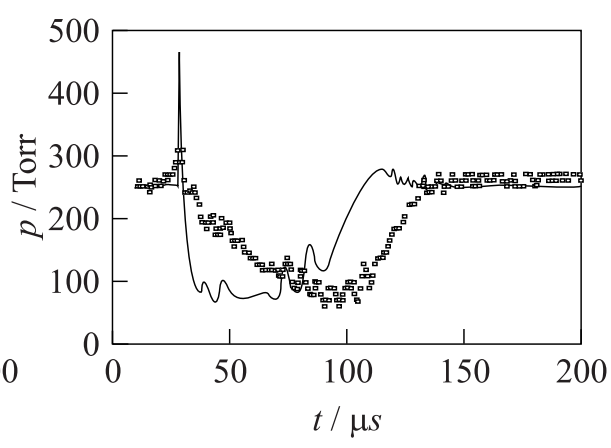

(b)

Figure 14 Surface pressure vs. time for hemisphere-cone cylinder [20]: (a) $n=0$; and $(b) n=1$. Signs refer to experiment and curves to computations

wavelength. The results are displayed in Fig. 14. The computed maximum pressure drop shows close agreement with experiment for both cases. However, the duration of the pressure disturbance for $n=0$ is significantly shorter than in the experiment. For $n=1$, the duration of the pressure disturbance shows closer agreement with experiment, although the initial pressure drop is substantially more rapid than in the experiment.

These results indicate the sensitivity of the computed flowfield to the imposed electric field pulse shape.

\section{CONCLUDING REMARKS}

Laser and microwave energy deposition have shown significant potential for flow control in high-speed flows. Substantial momentary decrease in drag can be obtained by pulsed laser or microwave discharge. Amelioration of potentially adverse shock-shock interactions can be also achieved.

Further research in modeling the laser and/or microwave discharge and its interaction with the flowfield around an aerodynamic body is needed.

\section{ACKNOWLEDGMENTS}

The research is supported by the U.S. Air Force Office of Scientific Research under Grant FA9550-10-1-0111 managed by Dr. John Schmisseur. 


\section{REFERENCES}

1. Knight, D., V. Kuchinskiy, A. Kuranov, and E. Sheikin. 2002. Aerodynamic flow control at high speed using energy deposition. 4th Workshop on Magneto-Plasma Aerodynamics for Aerospace Applications. Moscow: Institute for High Temperature, Russian Academy of Sciences.

2. Zheltovodov, A. 2002. Development of the studies on energy deposition for application to the problems of supersonic aerodynamics. Preprint No. 10-2002. Novosibirsk: Khristianovich Institute of Theoretical and Applied Mechanics.

3. Knight, D., V. Kuchinskiy, A. Kuranov, and E. Sheikin. 2003. Survey of aerodynamic flow control at high speed using energy addition. AIAA Paper No. 2003-0525.

4. Fomin, V., P. Tretyakov, and J.-P. Taran. 2004. Flow control using various plasma and aerodynamic approaches (short review). Aerospace Sci. Technol. 8:411-21.

5. Bletzinger, P., B. Ganguly, D. Van Wie, and A. Garscadden. 2005. Plasmas in high speed aerodynamics. J. Phys. D: Appl. Phys. 38:R33-R57.

6. Knight, D. 2008. Survey of aerodynamic drag reduction at high speed by energy deposition. J. Propul. Power. 24(6):1153-67.

7. Damon, E., and R. Tomlinson. 1963. Observation of ionization of gases by a ruby laser. Appl. Optics 2(5):546-47.

8. Minck, R. 1964. Optical frequency electrical discharges in gases. J. Appl. Phys. $35(1): 252-54$.

9. Raizer, Y. 1977. Laser-induced breakdown of gases. New York: Studies in Soviet Science, Consultants Bureau.

10. Adelgren, R., H. Yan, G. Elliott, D. Knight, T. Beutner, and A. Zheltovodov. 2003. Localized flow control by laser energy deposition applied to Edney IV shock impingement and intersecting shocks. AIAA Paper No. 2003-0031.

11. Kolesnichenko, Y. 2007. Microwave discharge in free space. In: Fundamentals of aerodynamic flow and combustion control by plasmas. EUCASS.

12. Knight, D., Y. Kolesnichenko, V. Brovkin, D. Khmara, V. Lashkov, and I. Mashek. 2009. Interaction of microwave-generated plasma with a hemisphere cylinder at Mach 2.1. AIAA J. 47(12):2996-3010.

13. Adelgren, R. 2002. Localized flow control with energy deposition. Ph.D. Thesis. New Brunswick, NJ, USA: Department of Mechanical and Aerospace Engineering, Rutgers University.

14. Adelgren, R., H. Yan, G. Elliott, D. Knight, T. Beutner, and A. Zheltovodov. 2005. Control of Edney IV interaction by pulsed laser energy deposition. AIAA J. 43(2):256-69.

15. Georgievsky, P., and V. Levin. 1993. Unsteady interaction of a sphere with atmospheric temperature inhomogeneity at supersonic speed. Mekhanika Zhidkosti Gaza 4:174-83. [In Russian.]

16. Edney, B. 1968. Anomalous heat transfer and pressure distributions on blunt bodies at hypersonic speeds in the presence of an impinging shock. Aeronautical Research Institute of Sweden. FAA Report 115. 
17. Van Wie, D., M. White, and G. Corpeling. 1990. NASp inlet design and testing issues. Johns Hopkins APL Technical Digest. 11(3-4).

18. Knight, D., Y. Kolesnichenko, V. Brovkin, D. Khmara, V. Lashkov, and I. Mashek. 2009. Interaction of a microwave-generated plasma with a blunt body at Mach 2.1. AIAA Paper No. 2009-0846.

19. Lashkov, V., I. Mashek, Y. Anisimov, V. Ivanov, Y. Kolesnichenko, M. Ryvkin, and A. Gorynya. 2004. Gas dynamic effect of microwave discharge on supersonic cone-shaped bodies. AIAA Paper No. 2004-0671.

20. Knight, D., Y. Kolesnichenko, V. Brovkin, V. Lashkov, and I. Mashek. 2010. Interaction of microwave-generated plasma with hemisphere-cone-cylinder. AIAA Paper No. 2010-1005. 